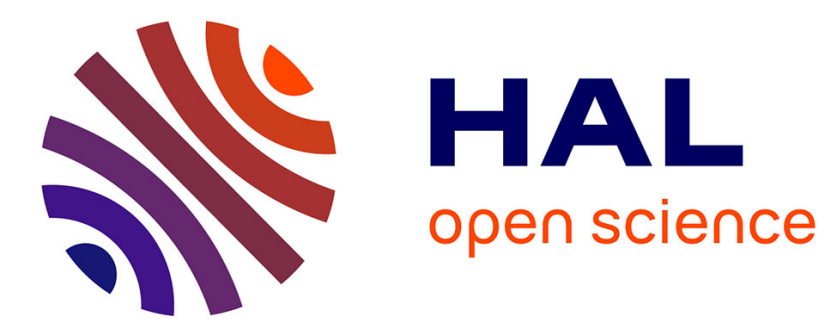

\title{
De l'animal expérimental au modèle en recherche biomédicale.
}

\author{
Gabriel Gachelin
}

\section{To cite this version:}

Gabriel Gachelin. De l'animal expérimental au modèle en recherche biomédicale.. 2008. halshs00334430

\section{HAL Id: halshs-00334430 \\ https://shs.hal.science/halshs-00334430}

Preprint submitted on 26 Oct 2008

HAL is a multi-disciplinary open access archive for the deposit and dissemination of scientific research documents, whether they are published or not. The documents may come from teaching and research institutions in France or abroad, or from public or private research centers.
L'archive ouverte pluridisciplinaire HAL, est destinée au dépôt et à la diffusion de documents scientifiques de niveau recherche, publiés ou non, émanant des établissements d'enseignement et de recherche français ou étrangers, des laboratoires publics ou privés. 


\title{
De l'animal expérimental au modèle en recherche biomédicale.
}

\author{
Gabriel Gachelin
}

Rehseis, UMR 7596 CNRS-Université Paris 7 Denis Diderot

Résumé. Depuis le début de l'expérimentation en biologie, les animaux sont utilisés pour étudier des phénomènes inabordables chez l'homme. Le développement depuis 1985, de procédures d'inactivation chez la souris de gènes suspects d'un rôle en pathologie humaine, a produit des souris dites « modèles de maladies humaines » avec l'implicite d'une identité des processus physiopathologiques entre homme et souris. Le passage du modèle pour.. au modèle de.. est discuté dans cet article ainsi que le retour nécessaire à une forme d'expérimentation sur l'homme pour tenir compte des spécificités de ce dernier.

Summary. Since the beginning of experimentation in biology, animals have been used to study processes that cannot be approached on human beings. The developement since 1985, of techniques aimed at precisely inactivationg mouse genes suspected to be involved in human diseases, has produced mice "model of human diseases », under the implicit assumption of an identity of physio-pathological processes in man and mouse. The passage from model for... to model of... is discussed in the present paper, along with the compulsory come back of a kind of experimentation on man, so as to take into account human specificities.

Plusieurs ouvrages collectifs ont abordé ces dernières années les divers contenus que les scientifiques donnent au mot « modèle $»^{1}$. Pour le seul cas des « modèles animaux en biologie et en médecine », le mot "modèle » y apparaît polysémique et recouvre des usages de l'animal expérimental dans des contextes épistémologiques très différents. L'opinion exprimée par A. Opinel, l'animal comme modèle est avant tout une métaphore de l'humain bien avant qu'il ne devienne animal de laboratoire résume bien le statut ambigu de l'animal ${ }^{2}$. Mais tout animal utilisé par un scientifique n'est pas en situation de devenir un modèle. L'étude de propriétés clairement spécifiques d'une espèce animale, situation dans laquelle ce que l'on étudie ne peut être que l'animal lui-même prototype d'une espèce, est une situation qui domine la zoologie. Elle est d'une certaine manière d'essence taxonomique. Elle peut

\footnotetext{
${ }^{1}$ P. Nouvel (dir) Enquête sur le concept de modèle, Presses universitaires de France, Paris, 2002; G. Gachelin (dir) Les organismes modèles dans la recherché médicale, Presses universitaires de France, Paris 2006; M. Armatte et A. Dahan (dir), "Modèles et modélisations", Revue d'bistoire des sciences, 2004, 57, 243-432; Collectif "The right organism for the job" Journal of History of Biology, 1993, 26, 253-367; Model organisms numéro special of The Scientist, 2003, 17 (supplément1) .

2 A. Opinel "Zoologie métaphorique" in G. Gachelin (dir) Les organismes modèles dans la recherché médicale, Presses universitaires de France, Paris 2006, pp 53-72
} 
cependant, du fait de particularités de l'animal étudié, déboucher sur un usage plus général de certains animaux, qui deviennent alors utilisés dans des séries d'expériences : d'observé pour lui-même, l'animal glisse au centre de l'expérimentation. Cette notion domine le $19^{\text {ème }}$ et le premier tiers du $20^{\text {ème }}$ siècles. La notion de «modèle animal » est quant à elle beaucoup plus récente et remonte, en tant que son usage est compris par tous sans avoir besoin de donner une définition, car l'expression est intuitivement comprise, à moins de 30 ans. L'utilisation de l'expression «animal modèle de maladie » ne devient quant à elle familière des revues scientifiques qu'à partir de 1980 environ $^{3}$ dans les faits avec l'apparition de la transgénèse animale et surtout celle de la recombinaison homologue chez la souris ${ }^{4}$.

La distinction entre animal expérimental et animal modèle n'est pas seulement historique : elle recouvre une approche différente du vivant. L'animal expérimental a, depuis le début de l'expérimentation en physiologie, été utilisé au laboratoire pour étudier commodément (et économiquement) des fonctions ou des propriétés que l'on ne pouvait pas expérimenter facilement et directement chez l'homme. La grenouille, petit martyr du laboratoire comme l'appelait Holmes ${ }^{5}$, a par exemple permis l'étude de l'influx nerveux et de la jonction neuromusculaire tout au long du $19^{\text {ème }}$ siècle, après avoir été utilisée par Spallanzani pour ses études sur la reproduction. Les neurophysiologistes la délaissent pour d'autres animaux quand des fonctions plus complexes ou plus intégrées que le réflexe de flexion, sont abordées, parce que la grenouille ne permet pas de répondre aux questions que l'on se pose : on conçoit qu'elle ne puisse pas permettre la vérification des conclusions de Broca ou de Sherrington. Elle ne prendra en fait valeur de modèle général que dans les seuls travaux pratiques des lycées, comme outil de démonstration des propriétés des réflexes et de la synapse neuromusculaire : il s'agit d'un modèle éducatif d'arc réflexe. Selon une formulation plus philosophique, l'exemple de la grenouille illustre la position de Rheinberger qui considère l'animal expérimental comme un objet épistémique': un instrument pour l'étude d'une maladie ou d'une fonction, qui ne possède de statut de modèle qu'aussi longtemps que la question que l'on pose au travers de son étude, n'a pas reçu de réponse. La réponse une fois obtenue, il disparaît en tant que tel. Cela ne lui interdit d'ailleurs pas de réapparaître en tant qu'outil pour l'étude de telle ou telle autre fonction, du fait de la masse de connaissances

\footnotetext{
${ }^{3} \mathrm{~J}$. Gayon, "les organismes modèles en biologie et medicine : introduction" in G. Gachelin (dir) Les organismes modèles dans la recherché médicale, Presses universitaires de France, Paris 2006, 9-44

${ }^{4}$ G. Gachelin "La construction de la Souris idéale" in G. Gachelin (dir) Les organismes modèles dans la recherché médicale, Presses universitaires de France, Paris 2006, 139-162

${ }^{5}$ F. L. Holmes. "The old martyr of science: the frog in experimental physiology". Journal of history of biology 1993, 26, 311-328.

${ }^{6}$ H.-J. Rheinberger, Towards a theory of epistemic things. Stanford University press, Stanford 1997
} 
accumulées à son propos et des avantages propres à l'espèce (facilité d'élevage, coût, longueur du cycle reproductif, accumulation de mutants etc). C'est le cas de la drosophile, dont le rôle moteur dans la naissance de la génétique formelle au début du $20^{\text {ème }}$ siècle avec Morgan et son école, s'estompe vers la seconde guerre mondiale au profit des outils et modèles de pensée de la génétique physiologique comme le champignon Neurospora puis le colibacille et ses phages, à l'origine du développement ce que l'on appellera à partir de 1935 la biologie moléculaire ${ }^{7}$ La drosophile réapparaît comme instrument d'étude du développement embryonnaire des insectes puis des vertébrés à partir de 1975 et les premiers travaux suggérant la nature des mutants homéotiques et vers 1990 pour l'étude de l'immunité innée. C'est le cas des amphibiens comme le Xénope, entre les mains de Spemann pour étudier vers 1930 la détermination de certaines étapes précoces du développement de l'œuf, plus précisément l'organisateur de Spemann ${ }^{8}$. C'est évidemment le cas de la souris, objet d'une véritable industrie, d'une idéologie de l'usage des souches génétiquement pures de mammifères et de modèle de maladies humaines ${ }^{9}$. Ces animaux expérimentaux ont été choisis pour de nombreuses raisons différentes, allant du coût économique au cycle reproductif en passant par sa génétique (c'est le cas de la drosophile et de la souris) la facilité d'expérimentation (parfois purement anatomique comme l'a souligné Claude Bernard). En pratique toutes les espèces animales ont été à un moment au moins utilisées au laboratoire, avant que ne soient sélectionnées sur des bases économiques et scientifiques, un plus petit nombre d'espèces particulières, schématiquement au début du $20^{\text {ème }}$ siècle, et qui sont devenues les habitants familiers des laboratoires.

L'usage d'animaux pour une expérimentation au laboratoire qui vise à décrire et comprendre une fonction biologique générale, a invariablement été sous-tendu par l'idée qu'il existait une grande unité des mécanismes physiologiques d'abord, ensuite des mécanismes moléculaires et informatifs à partir des années 1930 et la naissance de la biologie moléculaire associée à la génétique physiologique. On pouvait ainsi étudier l'influx nerveux chez les grenouilles et en inférer les propriétés générales de l'influx des nerfs humain, produire les cartes génétiques de la drosophile et établir celle de l'homme. L'animal, plus généralement l'être vivant expérimental, a donc été d'abord un instrument de la démonstration que ces mécanismes et

\footnotetext{
${ }^{7}$ M. Morange Histoire de la biologie moléculaire, La Découverte, Paris 1994

${ }^{8}$ J.-C. Dupont et S. Schmitt (dir) Du fenillet an gène [Texte imprimé] : une histoire de l'embryologie moderne, fin XVIIIe-XXe siècle Éd. Rue d'Ulm, Paris, 2003

9 J. -P. Gaudillière "Produire et utiliser les souris inbred, complexe biomedical, cancer et obésité aux Etats-Unis après 1945" in G. Gachelin (dir) Les organismes modèles dans la recherché médicale, Presses universitaires de France, Paris 2006, 163-180
} 
ces codes informatifs étaient essentiellement les mêmes d'une espèce à une autre, cette unité dissimulée par la diversité morphologique des espèces. De fait, du code génétique à la respiration cellulaire, les mécanismes et les codes sont bien partagés, à quelques exceptions près, par toutes les espèces vivantes. A l'intérieur de la classe des vertébrés, les grandes fonctions obéissent à des mécanismes et des codes également partagés: le développement d'un embryon de souris est essentiellement le même que celui de l'embryon humain et obéit à des mécanismes identiques. Mais cette capacité de généralisation va rapidement buter sur des phénomènes irréductibles les uns aux autres: les fonctions réalisées par le cerveau du rongeur ne seront largement pas les mêmes que chez le primate, bien que les mécanismes élémentaires à l'oeuvre (de circulation de l'influx, de transmission et de traitement de l'information) soient très essentiellement identiques. Il en est de même pour le système immunitaire, qui ne fonctionne pas exactement de la même manière chez tous les vertébrés. On conçoit pourquoi ces modèles d'étude de fonctions ont un statut d'objet épistémique, la limite est la réponse à la question posée, mais elle est aussi à trouver du côté des limites de la généralisation à partir d'une seule espèce animale. C'est d'ailleurs cette difficulté qui est minimisée en jonglant pour un même objet d'étude, par exemple l'immunité non spécifique, avec des organismes aussi apparemment différents que l'oursin, la drosophile, la souris et l'homme, jonglerie que permettent les techniques moléculaires modernes.

Le second usage de l'animal modèle est de façon dominante celui de modèle de maladies humaines. C'est le moment où l'animal passe du statut de modèle pour, pour prendre celui de modèle de, selon une distinction classique ${ }^{10}$, et presque invariablement compris dans une optique de transposition intégrale à l'homme des résultats obtenus sur lui. Cette affirmation d'homothétie, par exemple entre rongeurs et homme, s'est graduellement imposée à partir de la seconde guerre mondiale et constitue un mode de pensée important de la recherche en biologie. Peut-on substituer l'animal à l'homme pour comprendre la physiologie et la pathologie de ce dernier et transformer l'animal expérimental en véritable modèle de telle ou telle maladie ? Le point essentiel pour tenter de répondre à cette question gît précisément dans l'importance que l'on va attribuer aux détails « qui font la différence ». Ces détails (parfois assez notables comme le fonctionnement cérébral !) créent-ils suffisamment de différences entre espèces pour que l'on soit obligé de revenir dans l'expérimentation à une spécificité de l'espèce humaine, comme de toute autre espèce animale au demeurant? Au contraire, ces différences peuvent elles être éludées ? La réponse a apparemment changé dans le temps. Une

\footnotetext{
${ }^{10}$ E. Fox Keller,"Models of and models for: theory and practices in contemporary biology", Philosophy of science, 2000, 67.
} 
première réponse était donnée à la fin du $19^{\text {ème }}$ siècle. Par exemple, l'étude des maladies infectieuses qui se développe alors montre sans ambiguïté que pour les microbiologistes, l'homme est le vrai modèle d'une maladie humaine. Mais pour eux la maladie est celle d'un organisme considéré dans sa globalité. L'animal expérimental est indispensable, mais il est une sorte d'appareil de laboratoire intermédiaire entre la manipulation in vitro d'un microorganisme et l'observation de la maladie chez l'homme. L'animal, du moins celui chez qui l'agent infectieux peut croître, mime certaines étapes de la pathologie humaine et à permet de vérifier le rôle d'un microorganisme dans la pathogénie d'une infection, voire de purifier l'agent infectieux, mais il ne rend pas compte de l'infection dans la forme qu'elle prend chez l'homme. Derrière l'éreintement des travaux de Pasteur par Koch en 1882, la méthodologie et le code de bonne conduite expérimentale affirmés par ce dernier, ne représentent pas autre $\operatorname{chose}^{11}$. L'expérimentation sur l'homme représente, en cas de doute ou surtout en cas d'absence d'animal de laboratoire apte à mimer la maladie lorsqu'on y introduit l'agent infectieux suspecté, l'étape définitive d'une démonstration, la seule manipulation à prendre valeur de preuve : cela est particulièrement évident dans le cas des maladies à vecteurs comme la fièvre jaune. L'observation qu'il existe des corrélations entre présence de vecteurs infectés et maladies ne suffisait pas et l'expérimentation directe sur l'homme a été souvent une étape critique de l'affirmation du rôle d'un agent infectieux et de son vecteur. La démonstration apportée par Reed et par les chercheurs brésiliens en 1899 de la capacité d'un moustique à transmettre la fièvre jaune a donc été établie par l'expérimentation directe sur $1^{1}$ 'homme ${ }^{12}$. Le constat vaut également pour bien d'autres maladies parasitaires, en particulier les helminthiases au milieu du $19^{\text {ème }}$ siècle, et le paludisme jusque vers 1940. L'infection expérimentale délibérée de volontaires ( ?) a disparu ( ?), mais l'expérimentation sur des volontaires humains, de nos jours mieux encadrée, reste une étape clef dans la vérification de l'efficacité thérapeutique de telle ou telle molécule.

C'est précisément ce statut de non-identité homme/animal qui est implicitement récusé par l'expression «animal-modèle » telle qu'on l'utilise à partir des années 1980-1985. Elle implique en effet la capacité de reproduction chez la souris ou un autre animal modèle (en fait il en existe peu d'autres), de la totalité de qui se passe chez l'homme. Ces souris sont ellesmêmes une construction scientifique artificielle assez éloignée des populations naturelles de

\footnotetext{
${ }^{11}$ C. Gradman Krankheit im Labor. Robert Koch und die medizinische Bakteriologie, Göttingen: Wallstein-Verlag 2005 ,

${ }^{12}$ I. Löwy Virus, moustiques et modernité : Science, politique et la fièvre jaune au Brésil, Paris, Archives d'Histoire Contemporaine, 2001.
} 
souris $^{13}$. Il n'est pas dépourvu d'intérêt de noter que l'usage de l'expression survient après plusieurs décennies de mise en place de l'étude de divers mutants de souris, en cancérologie mais surtout à propos de l'obésité, comme substitut à l'étude biologique et comportementale sur l'homme. Gaudillière, travaillant sur les modèles murins de l'obésité et du cancer a clairement montré qu'une véritable industrie de production des souris et une politique de communication méthodique aient ancré dans le public l'idée que la souris était un modèle représentatif de l'humain au plan de la maladie et de certains comportements, alimentaires par exemple $^{14}$. Une notion en quelque sorte apurée d'animal-modèle de maladie se développe dans le cadre ouvert par la découverte que l'on peut modifier plus ou moins à volonté le génome des souris, et créer des mutations ciblées. Une forte partie de la recherche biomédicale s'oriente alors vers l'étude des effets de mutations crées précisément et à volonté par l'expérimentateur, qui affectent des gènes dont les produits, parce que mutés ou soumis à des régulations aberrantes, sont impliqués dans diverses maladies humaines. C'est à ce moment que l'on voit proliférer les articles intitulés « a mouse model of... », notion reprise par les pages scientifiques des grands journaux. Or ce que l'on note est que le modèle n'est en règle qu'une image imparfaite de la maladie humaine : la souris ne développe en général pas une maladie semblable à la maladie humaine. Au mieux, elle développe une forme murine qui en mime certaines étapes. Par exemple, l'inactivation d'un canal chlorure est à l'origine de la mucoviscidose chez l'homme. Que cette inactivation ne provoque essentiellement rien chez la souris n'empêche pas que cette souris mutée soit présentée comme modèle de la mucoviscidose et non pas comme modèle pour l'étude du canal chlorure, alors qu'elle peut effectivement se révéler utile pour l'étude des premières étapes de la mucoviscidose humaine, le test de médicaments et l'étude du fonctionnement de cette molécule indispensable à la compréhension de la cause initiale de la maladie. Le chercheur prend ainsi souvent la partie pour l'ensemble même si la discussion de l'article qui présente les résultats exprime, selon une rhétorique bien codifiée, les limites de l'interprétation et celles des espoirs de projection à l'homme. Il faut noter que ce choix de présentation accepté par le public est également d'une certaine manière la mise à l'écart du statut particulier de la maladie et du corps malade, qui possède par nature une dimension historique individuelle, au profit de la réduction de la maladie à une simple relation de cause à effet. Or, si la cause de la mucoviscidose est bien l'inactivation du canal Cl-, la maladie humaine, diverse dans ses manifestations comme elle

${ }_{13}^{13}$ G. Gachelin op.cit.

${ }^{14}$ I. Löwy and J.-P. Gaudillière «Disciplining cancer : mice and the practice of genetic purity » in J.-P.

Gaudillière and I. Löwy, The invisible industrialist :manufactures and the production of scientific knowledge, London, MacMillan, 1998 
peut l'être, lui est elle réductible? Aucun clinicien ne se hasardera à avancer cette proposition, surtout quand l'apparition et l'évolution de la maladie sont liés à l'histoire personnelle du sujet. Que dire d'une souris modèle de la schizophrénie parce que tel récepteur de neurotransmetteur a été inactivé... On retrouve ici par le biais de l'expérimentation sur des animaux pensés comme modèles, une autre manière de constater que l'homme reste le modèle de lui-même et que l'animal reste placé, sauf situation finalement assez peu fréquente, dans une dimension métaphorique par rapport à lui.

Cette spécificité d'une espèce par rapport à une autre, même proche au plan évolutif, n'est pas inattendue. En effet, chaque organisme est issu de l'assemblage de mécanismes et d'objets moléculaires par ailleurs relativement ubiquitaires : simplement, cette assemblage ajouté à la somme de petites différences dans la plupart des molécules, diffère d'une espèce à une autre. Ce constat est d'ailleurs une des questions des recherches sur l'évolution : comment faire du « différent» avec deux pools de gènes presque identiques ? A titre d'exemple, l'immunologie est largement fondée sur l'étude du système immunitaire des souris. La masse de connaissance acquise dans ce cadre est considérable. On peut s'en servir pour, par exemple, guérir les souris de la plupart de leurs maladies, comme leurs cancers. Mais la transposition à l'homme de ces procédures thérapeutiques s'est révélée le plus souvent illusoire: le système immunitaire de la souris n'a pas exactement les propriétés de celui de l'homme et le développement des cancers, pour ne prendre que cet exemple, n'utilise pas exactement les mêmes mécanismes chez la souris et chez l'homme. Les composants du système sont semblables, mais pas identiques et leur agencement ne produit pas précisément les mêmes effets dans les deux espèces. Les résultats obtenus dans une espèce comme la souris ne peuvent donc pas, en dépit de la généralité réelle des mécanismes mis en jeu, être facilement transposés à une autre et particulièrement à l'homme. Si l'on ajoute que le travail expérimental s'effectue sur des animaux génétiquement identiques, produits au laboratoire et jamais sélectionnés dans les conditions naturelles, et que de l'autre côté le polymorphisme génétique humain est considérable et qu'il s'y surimpose autant d'histoires individuelles qu'il y a de sujets, on conçoit que dans l'étude d'un processus physiopathologique même relativement simple comme une vaccination ou un tri de molécules thérapeutiques, on ne peut pas faire l'économie de l'étude sur des hommes ou des biopsies humaines. Bien entendu de nombreuses informations essentielles à la recherche médicale et à la biologie sont déduites de l'étude des animaux. Ce qui vient d'être écrit ne signifie évidemment pas la faillite des systèmes expérimentaux modèles en science. Il signifie simplement la nécessité de mettre l'accent sur la place de l'observation d'un processus physiopathologique et de 
l'expérimentation de ses certaines étapes chez l'homme, telles qu'elles semblent devenues possibles par les technologies analytiques avancées actuelles sans pour cela bousculer l'éthique, ce point étant à examiner indépendamment de ce propos.

Pourquoi donc, encore une fois, s'obstiner à présenter l'animal expérimental comme un modèle de l'humain ? Il existe plusieurs réponses possibles. Une réponse évidente est liée à la sociologie des institutions de recherche et à la nature même de la pratique de laboratoire, de la publication en biologie et de l'évaluation des chercheurs. Connaitre et étudier une sourismodèle de telle ou telle maladie permettra toujours d'observer quelque chose d'intéressant en soi et utile à l'étude de cette maladie chez l'homme. C'est également et peut être surtout l'assurance de publications dans de bons journaux. C'est en retour un élément important de carrière du chercheur et de financement du laboratoire. Cet aspect pragmatique, pour important qu'il soit, pour important que soit le cortège de biais dans la manière d'aborder un problème biomédical, ne doit cependant pas cacher le fait que l'emploi de l'animal-modèle comme structure homothétique de l'homme, précisément parce que le modèle est un variant génétique dans la plupart des cas, crée de facto un lien obligé entre structure génétique d'un sujet et sa maladie, dans une démarche qui vient compléter la chasse aux gènes de susceptibilité à ces mêmes maladies. On peut ainsi hasarder de manière un peu provocante, que cette réduction du langage schématisée par « la souris-modèle de », induit une réduction de la représentation de la maladie au seul produit de l'expression du génome du sujet malade. 